\title{
Thermodynamics of perfect fluids from scalar field theory
}

\author{
Guillermo Ballesteros, ${ }^{1,2, *}$ Denis Comelli, ${ }^{3, \dagger}$ and Luigi Pilo ${ }^{4,5, \sharp}$ \\ ${ }^{1}$ Institut de Physique Théorique, Université Paris Saclay, CEA, CNRS91191 Gif-sur-Yvette, France \\ ${ }^{2}$ CERN, Theory Division, 1211 Geneva, Switzerland \\ ${ }^{3}$ INFN, Sezione di Ferrara, 44124 Ferrara, Italy \\ ${ }^{4}$ Dipartimento di Scienze Fisiche e Chimiche, Università di L'Aquila, I-67010 L'Aquila, Italy \\ ${ }^{5}$ INFN, Laboratori Nazionali del Gran Sasso, I-67010 Assergi, Italy
}

(Received 1 June 2016; published 27 July 2016)

\begin{abstract}
The low-energy dynamics of relativistic continuous media is given by a shift-symmetric effective theory of four scalar fields. These scalars describe the embedding in spacetime of the medium and play the role of Stückelberg fields for spontaneously broken spatial and time translations. Perfect fluids are selected imposing a stronger symmetry group or reducing the field content to a single scalar. We explore the relation between the field theory description of perfect fluids to thermodynamics. By drawing the correspondence between the allowed operators at leading order in derivatives and the thermodynamic variables, we find that a complete thermodynamic picture requires the four Stückelberg fields. We show that thermodynamic stability plus the null-energy condition imply dynamical stability. We also argue that a consistent thermodynamic interpretation is not possible if any of the shift symmetries is explicitly broken.
\end{abstract}

DOI: 10.1103/PhysRevD.94.025034

\section{INTRODUCTION}

Fluid dynamics and thermodynamics are probably the oldest and better known examples of effective descriptions of a complicated underlying system in terms of a small number of macroscopic variables. Systems that admit a fluid description are found in nature at widely separate distance scales and energy regimes: from cosmological and astrophysical applications to heavy-ion physics and nonrelativistic condensed matter. A convenient formulation of fluid dynamics in the nondissipative limit is the pullback formalism—see [1] for a review-where a fluid is described through an ensemble of three derivatively coupled scalars that are interpreted as comoving coordinates of the fluid's elements. Within this formalism, fluid dynamics can be derived from an unconstrained action principle. A related approach was developed separately to obtain a field theory, symmetry driven, description of the fluctuations - sound waves-propagating in fluids and other types of continuous nonrelativistic media, see $[2,3]$. The relevant degrees of freedom from this point of view, which we can call phonons, can be identified with the Goldstone bosons of spontaneously broken translational symmetries in the pull-back formalism. Given this, the two approaches can be blended together into a fully relativistic

\footnotetext{
*guillermo.ballesteros@cea.fr comelli@fe.infn.it

*luigi.pilo@aquila.infn.it
}

Published by the American Physical Society under the terms of the Creative Commons Attribution 3.0 License. Further distribution of this work must maintain attribution to the author(s) and the published article's title, journal citation, and DOI. effective field theory (EFT) of continuous media [4,5], which turns to have an ample range of applications. In order to describe continuous media beyond anisotropic elastic solids, the field content of the pull-back formalism must be extended with a fourth scalar [5]. This allows to include superfluids in the picture and also more complex objects that are not (yet, maybe) found in nature, such as supersolids; see also [6] for other possible types of media. The fourth scalar can be interpreted as the carrier of an extra $U(1)$ charge [5] or as an internal time coordinate of the selfgravitating medium [7], offering a suggestive link to massive gravity theories and, in general, models of modified gravity; see $[4,7]$ and references therein.

In this work, we focus on perfect fluids, which correspond to two specific subclasses of the EFT of continuous media at leading order in derivatives (LO), as Fig. 1 illustrates. Although these systems can be considered the

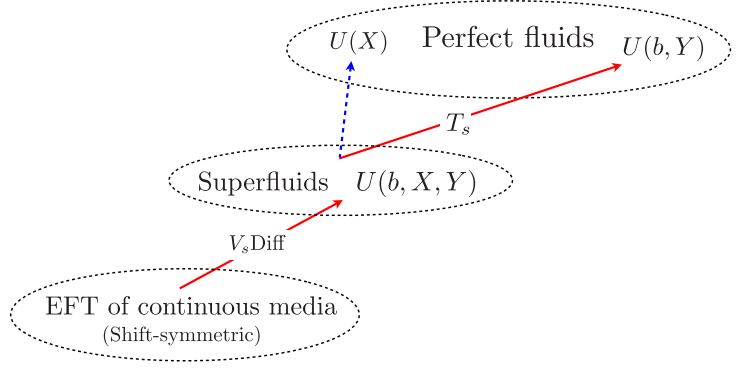

FIG. 1. Red continuous arrows represent the symmetries (4.2) and (4.7) leading to perfect fluids at leading order in derivatives in the EFT of nondissipative continuous media. The blue dashed arrow indicates that restricting the field content to a single (temporal) Stückelberg field leads to (irrotational) perfect fluids. 
simplest ones at the level of the energy-momentum tensor, they are not free of subtleties [5], and there is an ongoing effort towards understanding their properties in depth. Here we build upon the work of [5]—-see also [4]—where the thermodynamic interpretation of effective perfect fluids was studied. We extend the thermodynamic correspondences proposed in $[4,5]$, obtaining a thermodynamic dictionary that we have condensed in Table II.

Our analysis leads to the conclusion that a general thermodynamic picture (away from specific limits) requires indeed four scalar fields (instead of just three) and, consequently, implies an extension of the pull-back formalism. Remarkably, the form of the action required for such a thermodynamic picture is determined by a symmetry group that constitutes a specific set of continuous field redefinitions, selecting just two effective operators [5].

We show that a consistent thermodynamic interpretation requires, in any case, a shift symmetry for each field in the effective action. This is interesting because such symmetry is precisely the minimal requirement to have an EFT organized as a derivative expansion; see $[4,5,8,9]$. Moreover, shift symmetries are essential for the understanding of phonons - the degrees of freedom responsible for the propagation of sound-as Goldstone bosons [2,3] and also [10].

Finally, we argue that thermodynamic stability of perfect fluids plus the null-energy condition guarantee dynamical stability, i.e. the absence of ghost degrees of freedom and of exponential growth of fluctuations (around Minkowski spacetime). This holds true for all the types of perfect fluids allowed by the EFT.

Whereas the existence of an effective action description of nondissipative fluid dynamics should not be surprising, the fact that this action leads to a complete thermodynamic description of perfect fluids is remarkable and far reaching. Having a unified and general relativistic description of nondissipative dynamics and thermodynamics at the action level may open the possibility for novel applications of the pull-back formalism and the effective theory of fluids.

\section{THERMODYNAMICS IN A NUTSHELL}

Thermodynamics assumes that the equilibrium states of a simple system can be entirely characterized by the extensive variables volume, $V$, energy, $E$, and particle number of each species, $N_{i}$. In addition, it postulates the existence of a function of the extensive variables: the entropy, $S$, which is maximized in the evolution of the system. These two assumptions constitute the first and second postulates of thermodynamics. The third and fourth postulates establish, respectively, that in a composite system the entropy is an additive function over the constituent subsystems and that the entropy vanishes at zero temperature [11].

For our purposes, it is convenient to use intensive variables, defined by dividing the extensive variables over the volume. A simple scaling argument shows that $s=S / V$, the entropy density, is a function of the energy density, $\rho=E / V$, and the particle number density, $n=N / V$; namely, ${ }^{1}$

$$
s=s(\rho, n) \text {, }
$$

which constitutes the fundamental relation containing all the thermodynamic information of any simple system. Expressing this relation in the equivalent energy representation,

$$
\rho=\rho(s, n),
$$

and taking its differential, we get the first principle of thermodynamics:

$$
d \rho=T d s+\mu d n
$$

where the temperature and the chemical potential are defined as

$$
\left.T \equiv \frac{\partial \rho}{\partial s}\right|_{n},\left.\quad \mu \equiv \frac{\partial \rho}{\partial n}\right|_{s}
$$

From the additivity of the energy $E$ and the entropy $S$, the Euler relation follows,

$$
\rho+p=T s+\mu n,
$$

where $p$ is the intensive variable pressure:

$$
p=-\left.\frac{\partial E}{\partial V}\right|_{S, N} .
$$

The differential of the Euler relation, together with the first principle, leads to the fact the intensive variables $p, T$ and $\mu$ are not independent but satisfy the Gibbs-Duhem relation:

$$
d p=s d T+n d \mu
$$

Given two equations among (2.3), (2.5), and (2.7), the third follows.

From the definitions of the intensive variables $p, T$ and $\mu$, three equations of state can be written in the energy representation:

$$
T=T(s, n), \quad p=p(s, n), \quad \mu=\mu(s, n) .
$$

All together, these three equations of state are equivalent to the fundamental relation (2.2) or (2.1). Keeping only one or two of them among the three leads to some information

\footnotetext{
${ }^{1}$ In relativistic hydrodynamics, $n$ is usually meant to represent a charge density.
} 
TABLE I. Thermodynamic potentials and variables.

\begin{tabular}{lccc}
\hline \hline Thermodynamic potential & Independent variables & Legendre transf. to $\rho$ & Conjugate variables \\
\hline Energy density $\rho$ & $s, n$ & none & $T=\left.\frac{\partial \rho}{\partial s}\right|_{n}, \mu=\left.\frac{\partial \rho}{\partial n}\right|_{s}$ \\
Free-energy density $\mathcal{F}$ & $T, n$ & $\mathcal{F}=\rho-T s$ & $s=-\left.\frac{\partial \mathcal{F}}{\partial T}\right|_{n}, \mu=\left.\frac{\partial \mathcal{F}}{\partial n}\right|_{T}$ \\
Grand potential density $\omega$ & $T, \mu$ & $\omega=-p=\rho-T s-\mu n$ & $s=-\left.\frac{\partial \omega}{\partial T}\right|_{\mu}, n=-\left.\frac{\partial \omega}{\partial \mu}\right|_{T}$ \\
Potential density $\mathcal{I}$ & $s, \mu$ & $\mathcal{I}=\rho-\mu n$ & $T=\left.\frac{\partial \mathcal{I}}{\partial s}\right|_{\mu}, n=-\left.\frac{\partial \mathcal{I}}{\partial \mu}\right|_{s}$ \\
\hline \hline
\end{tabular}

about the system. Clearly, the equations of state can also be expressed in different ways depending on the two independent variables that are chosen. In general, given a simple system, two thermodynamic variables among $\{s, T, n, \mu, \rho, p\}$ can be taken as independent.

Starting from the energy representation (2.2), one can define other thermodynamic potentials, which are naturally associated to specific choices of pairs of independent variables different from $\{s, n\}$. For instance the GibbsDuhem relation (2.7) already tells us that we can use the pressure as a thermodynamic potential, which corresponds to (minus) the grand potential (density) $\omega$, i.e. $\omega=-p$. As (2.7) indicates, $\mu$ and $T$ are the associated independent variables in this case. We can also introduce the free-energy density $\mathcal{F}=\rho-T s$, which, using (2.3) and (2.5), satisfies $d \mathcal{F}=\mu d n-s d T$, effectively selecting $n$ and $T$. Similarly, we define another potential density-which bears no standard name $-\mathcal{I}=\rho-\mu n$ so $d \mathcal{I}=T d s-n d \mu$. These are all the potentials we will need for our purposes. The relations among them are summarized in Table I.

\section{ENERGY-MOMENTUM TENSOR AND CONSERVED CURRENTS OF PERFECT FLUIDS}

The energy momentum tensor (EMT), $T_{\mu \nu}$, and the currents of a macroscopically continuous system play a pivotal role in the determination of its thermodynamic interpretation (if any). Of particular relevance are the entropy and particle currents, $s^{\mu}$ and $n^{\mu}$, respectively. In agreement with the second postulate of thermodynamics,

$$
\nabla_{\mu} s^{\mu} \geq 0
$$

where the equality applies only for reversible (nondissipative) processes.

In this work, we focus on perfect fluids, which by the definition are the continuous media whose energymomentum tensor is of the form

$$
T_{\mu \nu}=(\rho+p) v_{\mu} v_{\nu}+p g_{\mu \nu}, \quad v^{\mu} v_{\mu}=-1,
$$

where the four-velocity of the fluid, $v_{\mu}$, is the (unique) timelike eigenvector with unit norm of $T_{\mu \nu}{ }^{2}$ For a perfect

\footnotetext{
${ }^{2}$ Throughout the paper, we use the metric signature $(-,+,+,+)$.
}

fluid, the entropy and the particle number currents are both parallel to $v_{\mu}$, and so we write

$$
n_{\mu}=n v_{\mu}, \quad s_{\mu}=s v_{\mu} .
$$

By using Eq. (2.5), the perfect fluid EMT can be expressed as

$$
T_{\mu \nu}=\left(T s_{\mu}+\mu n_{\mu}\right) v_{\nu}+p g_{\mu \nu} .
$$

Applying $^{3}$ the conservation of the EMT to (3.4), the projection $v^{\mu} \nabla^{\nu} T_{\mu \nu}=0$ leads to

$$
T \nabla^{\alpha} s_{\alpha}+\mu \nabla^{\alpha} n_{\alpha}=0
$$

where we have used the Gibbs-Duhem relation (2.7) along the fluid flow $p^{\prime}=n \mu^{\prime}+s T^{\prime}$, denoting by $f^{\prime}=v^{\mu} \nabla_{\mu} f$ the Lie derivative of a function $f$ along the four-velocity of the fluid. The equation (3.5) is equivalent to the first principle of thermodynamics, and it tells us that for a perfect fluid (with nonvanishing chemical potential, $\mu$ ) a change in the particle current implies a variation of the entropy current. If the particle number current is conserved (or if $\mu$ is negligible), the entropy current is also conserved and we can write $s^{\prime}+\theta s=0$, where $\theta=\nabla^{\mu} v_{\mu}$ is the expansion. In particular, if $\nabla^{\mu} n_{\mu}=0$, the equation $n^{\prime}+\theta n=0$ also holds and we find that the entropy per particle $\sigma=s / n$ is conserved along the flow lines: $\sigma^{\prime}=0$, thus defining an adiabatic fluid. Instead, an isentropic fluid is defined as one that has constant entropy per particle, i.e. $\nabla_{\mu} \sigma=0$.

Using $h^{\mu \nu}=g^{\mu \nu}+v^{\mu} v^{\nu}$ to project the conservation of the EMT orthogonally to $u_{\mu}$, we get the Euler equations for a perfect fluid,

$$
(p+\rho) a_{\mu}+h_{\mu}^{\nu} \nabla_{\nu} p=0
$$

where $(p+\rho) / n$ is the enthalpy per particle and $a_{\mu}=$ $v^{\nu} \nabla_{\nu} v_{\mu}$ is the acceleration along the flow lines. This equation manifestly shows that the acceleration $a_{\mu}$ depends on the pressure gradient, as expected, since $\rho+p$ is the relativistic generalization of the mass density.

\footnotetext{
${ }^{3}$ We thank S. Matarrese for pointing out the paper [10] in which a similar treatment to the one in this section is given.
} 
Defining the vorticity tensor as (see e.g. [12])

$$
\Omega_{\mu \nu}=\left(\nabla_{\nu} w_{\mu}-\nabla_{\mu} w_{\nu}\right)
$$

from the enthalpy current $w^{\mu}=(\rho+p) / n v^{\mu}$, we can express the conservation of the EMT in terms of the Carter-Lichnerowicz equations:

$$
n \Omega_{\alpha \nu} v^{\nu}=n T \nabla_{\alpha} \sigma-w_{\alpha} \nabla^{\nu} n_{\nu}
$$

Indeed, (3.5) and (3.6) come from projecting (3.8) along $v^{\mu}$ and orthogonally to it.

A perfect fluid is sometimes said to be irrotational if $\Omega_{\mu \nu}=0$. In this sense, an irrotational perfect fluid with $\nabla^{\mu} n_{\mu}=0$ satisfies $\nabla_{\mu} \sigma=0$ and is called isentropic. Clearly, a fluid that is adiabatic $\left(\sigma^{\prime}=0\right)$ is not necessarily irrotational in the previous sense.

It is also common usage to call irrotational a perfect fluid whose four-velocity is the derivative of a scalar quantity, i.e. $v_{\mu}=\partial_{\mu} \Psi$. Clearly, this notion is not equivalent, in general, to $\Omega_{\mu \nu}=0$.

\section{EFFECTIVE ACTION FOR NONDISSIPATIVE HYDRODYNAMICS}

The study of relativistic fluid dynamics from an unconstrained action principle has a long history. In this work, we will closely follow the treatment of the subject that we gave in [7]. Our approach is related to Carter's geometrical formulation of the problem [13], but embedded in an effective field theory (EFT) framework; as proposed e.g. in [2,3] and later in [4]; see also [5]. Various aspects and applications of the EFT approach for describing continuous media have been developed in [4-6,8,9,14-23]. Interestingly, this framework can be used, for instance, to treat massive and modified gravity in a unified way, interpreting them as self-gravitating media; see [7]. ${ }^{4}$ For the development of Carter's idea into the subsequent pull-back formalism, see [27,28]. A review of this formalism is given in [1] and recent applications in the context of cosmology can be found in $[7,9,17,18,21,29-34]$. The idea that lies at the core of this treatment consists in using the Lagrangian coordinates of a continuous medium as the low-energy degrees of freedom of the EFT. In the original pull-back formalism, three scalar fields $\Phi^{a}, a=1,2,3$, identify the fluid elements as they propagate in space. Here we add a fourth scalar $\Phi^{0}$, which may be interpreted at this stage as an internal time coordinate of the medium. Indeed, these scalars can be seen as Stückelberg fields restoring broken diffeomorphisms in four-dimensional spacetimes; see e.g. [7,24-26].

\footnotetext{
${ }^{4}$ See also [24-26] for previous works on massive gravity using the Stückelberg "trick."
}

We require that the action of these four fields respects the shift symmetries

$\Phi^{A} \rightarrow \Phi^{A}+f^{A}, \quad \partial_{\mu} f^{A}=0, \quad A, \mu=0,1,2,3$.

In addition, we impose invariance under internal spatial volume preserving diffeomorphisms

$$
\begin{gathered}
V_{s} \text { Diff }: \Phi^{a} \rightarrow \Psi^{a}\left(\Phi^{b}\right), \quad \operatorname{det}\left(\frac{\partial \Psi^{a}}{\partial \Phi^{b}}\right)=1, \\
a, b=1,2,3 .
\end{gathered}
$$

With these symmetries, at leading order in the derivative expansion (LO), there are only two timelike independent four-vectors and just three independent scalar operators, which can be chosen to be

$u^{\mu}=-\frac{\epsilon^{\mu \alpha \beta \gamma}}{6 b \sqrt{-g}} \epsilon_{a b c} \partial_{\alpha} \Phi^{a} \partial_{\beta} \Phi^{b} \partial_{\gamma} \Phi^{c}, \quad \mathcal{V}^{\mu}=-\frac{\partial^{\mu} \Phi^{0}}{\sqrt{-X}}$

and

$b=\sqrt{\operatorname{det} \boldsymbol{B}}, \quad X=\partial_{\mu} \Phi^{0} \partial^{\mu} \Phi^{0}, \quad Y=u^{\mu} \partial_{\mu} \Phi^{0}$,

where the four-vectors have norm -1 and $\boldsymbol{B}$ is the $3 \times 3$ matrix of components $B^{a b}=\partial^{\mu} \Phi^{a} \partial_{\mu} \Phi^{b}$. Then, the LO action including gravity ${ }^{5}$ is $[5,16]$ (see also [7])

$$
S=M_{\mathrm{pl}}^{2} \int d^{4} x \sqrt{-g} R+\int d^{4} x \sqrt{-g} U(b, Y, X),
$$

where $M_{\mathrm{pl}}^{2}=1 /(16 \pi G)$, with $G$ being Newton's constant. The gravitational EMT tensor is

$T_{\mu \nu}=\left(U-b U_{b}\right) g_{\mu \nu}+\left(Y U_{Y}-b U_{b}\right) u_{\mu} u_{\nu}+2 X U_{X} \mathcal{V}_{\mu} \mathcal{V}_{\nu}$,

where $U$ is an arbitrary master function and we have denoted by $U_{b}, U_{X}$, and $U_{Y}$ its partial derivatives. Since $u^{\mu}$ and $\mathcal{V}^{\mu}$ are, in general, not parallel, this EMT does not describe a perfect fluid like (3.2). Actually, (4.6) was proposed as the EMT of a superfluid; see $[35,36]$ (and also e.g. $[16,28]$ within the framework we use). Indeed, since $\mathcal{V}_{\mu} \sim \partial_{\mu} \Phi^{0}$ is irrotational, it has been associated to the intrinsic superfluid component of the medium, whereas $u_{\mu}$ would correspond to the standard fluid component.

In this work, we will be solely interested in perfect fluids, which can be obtained either from $u^{\mu}$ or $\mathcal{V}^{\mu}$ using the action (4.5), as the EMT (4.6) shows neatly. Concretely, if $U=$ $U(b, Y)$ or $U=U(X)$, the resulting medium is a perfect

\footnotetext{
${ }^{5}$ As explained in [9], the Einstein-Hilbert term is the unique possible choice at this order in derivatives.
} 
fluid. Remarkably, the first of these two cases is obtained requiring that the action has to be invariant under [5] (see also e.g. $[7,8,16])$

$$
T_{s}: \Phi^{0} \rightarrow \Phi^{0}+f\left(\Phi^{a}\right), \quad a=1,2,3,
$$

with $f$ being an arbitrary function. Clearly, $U(b)$ and $U(Y)$ also describe perfect fluids. Assuming that the action depends only on the spatial Stückelbergs $\Phi^{a}, a=1,2$, 3 , the only possibility respecting $V_{s}$ Diff is $U(b)$. Similarly, $U(X)$ is the only possibility if the action contains only $\Phi^{0}$ and respects a shift symmetry. These two types of perfect fluids, $U(b)$ and $U(X)$, are manifestly different from each other since $\mathcal{V}^{\mu}$ is irrotational-in the sense that it is the gradient of the scalar $\Phi^{0}$ - and $u^{\mu}$ is not.

At higher orders in the derivative expansion, the symmetries we have discussed do not protect the perfect fluid form of the EMT of these systems, which generically acquires other terms; see e.g. [5,9,19]. This means that from the point of view of the EFT, the "perfectness" of perfect fluids is only an approximate low-energy feature.

\section{A. Conserved currents of effective perfect fluids}

The different kinds of perfect fluids that can be obtained out of the general EMT (4.6) can be classified according to their conserved currents; see also [7]. These currents are of two types: Noether currents and currents that are conserved independently of any symmetry of the action. In what follows when we say that a current is conserved we mean that it is covariantly conserved, i.e. $\nabla_{\mu} J^{\mu}=0$.

(i) $U(b)$ has currents of two types. First, there is

$$
\mathcal{J}^{\mu}=b u^{\mu}
$$

which is conserved off-shell. Actually, any current of the form $f\left(\Phi^{a}\right) \mathcal{J}^{\mu}$, where $f$ is a function of the fields $\Phi^{a}$, is also conserved thanks to $u^{\mu} \partial_{\mu} \Phi^{a}=0$. This last equation shows that $\Phi^{a}$ are actual comoving coordinates of the fluid, as the pull-back formalism requires.

(ii) $U(X)$ has only one independent conserved current,

$$
\mathcal{X}^{\mu}=-2 \sqrt{-X} U_{X} V^{\mu}
$$

coming from the symmetry $\Phi^{0} \rightarrow \Phi^{0}+c^{0}, \partial_{\mu} c^{0}=0$, and giving the dynamics of $\Phi^{0}$ for $U(X)$. The factor 2 is included for later convenience.

(iii) $U(Y)$ has, in addition to the same currents as $U(b)$, the current

$$
\mathcal{Y}^{\mu}=U_{Y} u^{\mu} .
$$

As a matter of fact, any $f\left(\Phi^{a}\right) \mathcal{Y}^{\mu}$ is conserved as well. These currents are due to the symmetry $T_{s}: \Phi^{0} \rightarrow \Phi^{0}+f\left(\Phi^{a}\right)$. (iv) $U(b, Y)$ has the same currents as $U(Y)$ because they share field content and symmetries. We recall that $U(b, Y)$ is the most general case at $\mathrm{LO}$ assuming that the action is symmetric under $V_{s}$ Diff and $T_{s}$.

As we will see in Sec. V, only the currents $\mathcal{J}^{\mu}, \mathcal{Y}^{\mu}$, and $\mathcal{X}^{\mu}$ are needed to establish the full thermodynamic dictionary. Actually, other conserved currents are present (depending on the operator content) but are not relevant for our purposes in this work; for a discussion, see for instance $[4,5,7,15,18]$. Requiring only the symmetry $V_{s}$ Diff and the shift symmetry (4.1) for $\Phi^{0}$ the action is (4.5) and the EMT is not a perfect fluid. In this case, the conserved current associated to the second of these symmetries is precisely the sum of the currents (4.9) and (4.10): $\mathcal{X}^{\mu}+\mathcal{Y}^{\mu}$. As before, any current of the form $\left(\mathcal{X}^{\mu}+\mathcal{Y}^{\mu}\right) f\left(\Phi^{a}\right)$ is also conserved, by virtue of $u^{\mu} \partial_{\mu} \Phi^{a}=0$.

\section{B. Energy density and pressure of perfect fluids}

Barotropic fluids are common in several branches of physics; in particular in cosmology. They serve to model in a first and crude approximation the basic matter species of the $\Lambda \mathrm{CDM}$ model: cold dark matter (CDM), baryons, photons and neutrinos. The current accelerated expansion can also be modeled with a barotropic fluid, be it a cosmological constant, $\Lambda$, or a more exotic component with a sufficiently negative equation of state $w=p / \rho$. The usual definition is that barotropic fluids are those whose pressure is a function of the energy density alone, i.e. $p=p(\rho)$. This can be generalized by defining a barotropic fluid as one whose pressure is completely characterized knowing the energy density at each point of the fluid (or vice versa) [18]. In this sense, the perfect fluids $U(X)$, $U(b)$, and $U(Y)$ are all barotropic. Indeed, in these cases, the pressure and the energy density are related, respectively, through the relations

$$
\begin{aligned}
\text { (i) } \rho & =-U(b), & & p=U-b U_{b}, \\
\text { (ii) } p & =U(X), & & \rho=2 X U_{X}-U, \\
\text { (iii) } p & =U(Y), & & \rho=Y U_{Y}-U .
\end{aligned}
$$

The perfect fluids $U(b, Y)$ are not barotropic, in general, simply because their Lagrangians are functionals of two independent operators:

(iv) $p=U-b U_{b} \quad$ and $\quad \rho=Y U_{Y}-U, \quad U=U(b, Y)$.

The relation between pressure and energy density for $U=$ $U(b, Y)$ can be determined only if another thermodynamic quantity is also known. However, there exist specific choices of the function $U(b, Y)$ that are barotropic. In particular, a constant $w=p / \rho$ can be obtained for $U(b, Y)=b^{1+w} \mathcal{U}\left(Y b^{-w}\right)$, with $\mathcal{U}$ being an arbitrary 
function. Another possibility is $U(b, Y)=b^{1+w}+\lambda Y^{1+1 / w}$, with constant $\lambda$. A constant equation of state can also be reproduced with $U(X)=X^{(1+1 / w) / 2}$. Nonrelativistic matter with $p=0$, such as standard CDM, admits only the description $U(b)=b$.

There are also other examples of perfect barotropic fluids that have been used often in astrophysics and cosmology, and can be easily described within our framework. For instance, a Chaplygin gas satisfies $p=-A / \rho$, with constant $A$, and can be obtained from $U(b)=\sqrt{A+\lambda b^{2}}$, $U(Y)=\sqrt{A+\lambda Y^{2}}$ or $U(X)=\sqrt{A+\lambda X}$, with constant $\lambda$.

\section{THERMODYNAMIC DICTIONARY FOR PERFECT FLUIDS}

In this section, we give the thermodynamic interpretation of the EFT of perfect fluids described in Sec. IV by giving the correspondence between the thermodynamic variables and the operators $b, Y$, and $X$ for the different kinds of perfect fluids. These results extend the interpretations that were previously proposed in $[4,5]$.

For a simple thermodynamic system, among the six variables $(s, T, n, \mu, \rho, p)$, two of them can be taken as independent, say $z_{1}$ and $z_{2}$, and the remaining four can be expressed in terms of $z_{1}$ and $z_{2}$. The EMT provides the explicit form of $p$ and $\rho$; see (4.11)-(4.12). Our outcome, once the independent variables have been chosen, is that $U$ is proportional to the natural thermodynamical potential associated with such thermodynamical variables; see Table I. In the Appendix, we describe a method based on the counting of derivatives of $U$ with respect to the EFT operators, showing that this is indeed the case.

Let us start with the fluid described by $U(b, Y)$, which depends on two operators, and choose as independent thermodynamic variables $(T, n)$. From (4.12), we have that $\rho=Y U_{Y}-U$ and $p=U-b U_{b}$. If a thermodynamic interpretation exists, the operators $b$ and $Y$ of the scalar field theory should be functions of the chosen thermodynamic variables, namely, $Y=Y(T, n)$ and $b=b(T, n)$. From Table I, it is clear that the natural thermodynamic potential with variables $(T, n)$ is the free-energy density $\mathcal{F}$, which is obtained from $\rho$ or $p$ after a single Legendre transformation. The natural choice is to take $U(b(n, T)$, $Y(n, T))=-\mathcal{F}(n, T)$ and find for which $b=b(n, T)$ and $Y=Y(n, T)$ the fundamental thermodynamic relations (2.3) and (2.5) are satisfied. There are multiple ways in which this can be done, all of them leading to the same set of solutions. Using that $d \mathcal{F}=\mu d n-s d T$-see Table Ithe Euler relation (2.5) becomes $b \mathcal{F}_{b}-Y \mathcal{F}_{Y}=n \mathcal{F}_{n}-$ $T \mathcal{F}_{T}$. Expressing the derivatives with respect to $b$ and $n$ in terms of derivatives with respect to $T$ and $n$ and imposing that the resulting equation has to be valid for all $\mathcal{F}$, we obtain $b=n b_{n}-T b_{T}$ and $Y=T Y_{T}-n Y_{n}$. We can now use the Gibbs-Duhem relation (2.7) or, simply, the definition $\mathcal{F}=\rho-T s$. Either way, we are led to $T \propto Y, n \propto b$, $s \propto U_{Y}$ and $\mu \propto U_{b}$; see Table II. An analogous procedure can be followed when the thermodynamical variables $(s, \mu)$ are used, in this case the natural thermodynamic potential is $\mathcal{I}$ and one can check that setting $U(b(s, \mu), Y(s, \mu))=$ $-\mathcal{I}(s, \mu)$ all the thermodynamic relations are satisfied if $b=s$ and $Y=T$; the result is also given in Table II. The possibility of identifying $-U(b, Y)$ as the thermodynamic potential $\mathcal{I}(s, \mu)$ was already found in [5].

The dictionary of Table II shows that some combinations of operator content and independent thermodynamic variables have no entry. By looking at Table II, for the case $U(b, Y)$, note that in the dictionary there is no (direct) entry for some choice of independent thermodynamical variables. This is the case for the choice of variables $(\mu, T)$ and $(n, s)$ for $U(b, Y)$. The reason is that the EMT tensor for $U(b, Y)$ is such that $U$ is not proportional to $p=-\omega$ or to $\rho$ as thermodynamics should require (see Table I) if $U$ is interpreted to be the corresponding thermodynamical potential. As it was already argued in [5], $U(b, Y)$ can be identified with $-I(s, \mu)$ because this potential can be obtained from $\rho(n, s)$ using a Legendre transformation; see Table I. The identification of $U(b, Y)$ with $-\mathcal{F}(n, T)$ can be argued on the same ground.

It is important to stress that the nonexistence of an entry in the dictionary for some variables does not mean that it is impossible to use those variable. Take, for instance, the entries relative to $U(b, Y)$ and variables $(n, T)$. By using a Legendre transformation, one can always switch from $(n, T)$ to $(n, s)$, still getting $T=Y$ and $\mu=-U_{b}$. Thus, the entries in the dictionary correspond to inequivalent thermodynamic interpretations of the very same scalar field action.

Let us now consider the Lagrangian $U(b)$. In this case given the fact that at least two independent thermodynamical variables are needed to describe a simple system and a single operator is present in the action, it is not surprising that various possibilities exist. Using the Euler relation (2.5) and the expressions (4.11) for the energy density and pressure, we get $\mu n+T s=-b U_{b}$. In addition, the GibbsDuhem equation (2.7) tells us that $s d T+n d \mu=-b U_{b b} d b$, and the first principle of thermodynamics (2.3) is $T d s+\mu d n=-U_{b} d b$. To proceed further, we can choose $n$ and $T$ as our two independent thermodynamic variables, so that $b=b(n, T)$. It is easy to check that $n=b$ and $\mu=$ $-U_{b}$ solve these equations for all $U_{b}$. This implies that $s=0$ and, according to the fourth postulate of Sec. II, $T=0$. Therefore, $U(b)$ can describe a perfect fluid in the limit of zero temperature. With this interpretation, that was already proposed in [4], the current $\mathcal{J}^{\mu}$ defined in (4.8) plays the role of the conserved particle current, and the entropy current vanishes in this limit.

Let us consider now as independent thermodynamic variables $(\mu, T)$, i.e. we assume $b=b(\mu, T)$. It is easy to see that $b=s$ and $T=-U_{b}$ can solve the thermodynamic equations. For consistency, this implies that $n$ must be zero. 
TABLE II. The EFT-thermodynamics dictionary for perfect fluids. The first row gives the energy density, the pressure and the relevant currents for the thermodynamic interpretation of each type of perfect fluid. The other rows give the entries of the dictionary for the four pairs of independent thermodynamic variables and their corresponding potentials. When it appears, $f$ is an undetermined function (and $f^{\prime}$ is its derivative) of the argument $z=\mu / T$ or $\sigma=s / n$.

\begin{tabular}{|c|c|c|c|c|}
\hline & $\begin{array}{c}\boldsymbol{U}(\boldsymbol{b}) \\
\rho=-U \\
p=U-b U_{b} \\
\mathcal{J}_{\mu}=b u_{\mu}\end{array}$ & $\begin{array}{c}\boldsymbol{U}(\boldsymbol{Y}) \\
\rho=-U+Y U_{Y} \\
p=U \\
\mathcal{Y}_{\mu}=U_{Y} u_{\mu}\end{array}$ & $\begin{array}{c}\boldsymbol{U}(\boldsymbol{X}) \\
\rho=-U+2 X U_{X} \\
p=U \\
\mathcal{X}_{\mu}=-2(-X)^{1 / 2} U_{X} \mathcal{V}_{\mu}\end{array}$ & $\begin{array}{c}\boldsymbol{U}(\boldsymbol{b}, \boldsymbol{Y}) \\
\rho=-U+Y U_{Y} \\
p=U-b U_{b} \\
\mathcal{J}_{\mu}=b u_{\mu}, \mathcal{Y}_{\mu}=U_{Y} u_{\mu}\end{array}$ \\
\hline $\begin{array}{l}(\boldsymbol{\mu}, s) \\
\mathcal{I}=-U\end{array}$ & $\begin{array}{c}b=s \\
n=0 \\
T=-U_{b} \\
\mathcal{J}_{\mu}=s u_{\mu}\end{array}$ & $\begin{array}{c}Y=\mu \\
n=U_{Y} \\
T=0 \\
\mathcal{Y}_{\mu}=n u_{\mu}\end{array}$ & $\begin{array}{c}X=-\mu^{2} \\
n=-2 U_{X} \sqrt{-X} \\
T=0, \mathcal{X}_{\mu}=-n / 2 \mathcal{V}_{\mu} \\
\mathcal{X}_{\mu}=n \mathcal{V}_{\mu}\end{array}$ & $\begin{array}{c}b=s, Y=\mu \\
n=U_{Y} \\
T=-U_{b} \\
\mathcal{J}_{\mu}=s u_{\mu}, \mathcal{Y}_{\mu}=n u_{\mu}\end{array}$ \\
\hline $\begin{array}{l}(\boldsymbol{n}, \boldsymbol{T}) \\
\mathcal{F}=-U\end{array}$ & $\begin{array}{c}b=n \\
s=0 \\
\mu=-U_{b} \\
\mathcal{J}_{\mu}=n u_{\mu}\end{array}$ & $\begin{array}{c}Y=T \\
s=U_{Y} \\
\mu=0 \\
\mathcal{Y}_{\mu}=s u_{\mu}\end{array}$ & $\begin{array}{c}X=-T^{2} \\
s=-2 U_{X} \sqrt{-X} \\
\mu=0 \\
\mathcal{X}_{\mu}=s \mathcal{V}_{\mu}\end{array}$ & $\begin{array}{c}b=n, Y=T \\
s=U_{Y} \\
\mu=-U_{b} \\
\mathcal{J}_{\mu}=n u_{\mu}, \mathcal{Y}_{\mu}=s u_{\mu}\end{array}$ \\
\hline $\begin{array}{l}(\boldsymbol{\mu}, \boldsymbol{T}) \\
\omega=-U \\
z=\mu / T\end{array}$ & & $\begin{array}{c}Y=T f(z) \\
s=U_{Y}\left(f-z f^{\prime}\right) \\
n=f^{\prime} U_{Y} \\
T f \mathcal{Y}_{\mu}=(\rho+p) u_{\mu}\end{array}$ & $\begin{array}{c}X=-T^{2} f(z) \\
s=U_{X}\left(\mu f^{\prime}-2 T f\right) \\
n=-U_{X} T f^{\prime} \\
T f^{1 / 2} \mathcal{X}_{\mu}=(\rho+p) \mathcal{V}_{\mu}\end{array}$ & \\
\hline $\begin{array}{l}(\boldsymbol{n}, \boldsymbol{s}) \\
\rho=-U \\
\sigma=s / n\end{array}$ & $\begin{array}{c}b=s f\left(\sigma^{-1}\right) \\
\mu=-U_{b} f^{\prime} \\
T=-U_{b}\left(f-f^{\prime} / \sigma\right) \\
\mathcal{J}_{\mu}=s f u_{\mu}\end{array}$ & & & \\
\hline
\end{tabular}

According to the discussion in Sec. II, the choice $n=0$ should be understood as zero particle density. However, in relativistic hydrodynamics, $n$ is usually meant to represent a charge density, making this point of view more appealing. In this case, $b=s$ and the current $\mathcal{J}^{\mu}$ represents the entropy current. This is the interpretation that was earlier advocated in [5] and subsequent works.

It is interesting to note that there are choices of independent thermodynamic variables for which an arbitrary function of their ratio appears in the dictionaries of $U(b)$. Consider, as an example, $U(b)$ and take $n$ and $s$ as independent variables, so that the natural thermodynamic potential is the energy density, $\rho(n, s)$. The equation $\rho+$ $U=0$ is automatically satisfied, whereas the Euler relation becomes $b=n b_{n}+s b_{s}$, whose general solution is $b=s f(n / s)$, with $f$ being an unspecified function of $\sigma^{-1}=n / s$. The conservation of the current $\mathcal{J}^{\mu}=b u^{\mu}$ gives $(\rho+p) \theta=-T s^{\prime}-s T^{\prime}$, which is the first principle of thermodynamics in the form (3.5). Similarly, for $U(X)$ and $U(Y)$ with $\mu$ and $T$ as independent thermodynamic variables, the corresponding entries of the dictionary depend on the ratio $z=\mu / T$. An interesting discussion of the case $U(X)$ can be found in [10]. When an unspecified function $f$ is found, it also enters in the conserved currents and their physical interpretation is more subtle.

Notice that the entries for $U(b)$ and $U(Y)$ relative to the variables $(\mu, s)$ and $(n, T)$ represent also the limiting cases of $U(b, Y)$ for $U_{Y}=0$ and $U_{b}=0$, respectively. In addition, the limits for $(\mu, s)$ and $(n, T)$ can be obtained choosing specific functions $f$ for the fluids that depend on a single operator. Concretely, this can be done choosing $f$ to be a constant or, imposing $f=n / s$ for $U(b) ; f=\mu / T$ for $U(Y)$ and $f=\mu^{2} / T^{2}$ for $U(X)$.

The analysis of this section leads to the conclusion that $U(b, Y)$ arises as the most appropriate Lagrangian for a complete thermodynamic description of a relativistic perfect fluid. Having two effective operators allows a full matching to the thermodynamic relations describing a simple system. This is translated into the fact that for $U(b, Y)$, the currents $\mathcal{J}^{\mu}$ and $\mathcal{Y}^{\mu}$ can be put in correspondence with the entropy and particle currents-both of which are conserved-giving an adiabatic fluid; see Sec. II. The Lagrangian $U(b, Y)$ was already identified in [5] as the most general one for a perfect fluid carrying a conserved charge. Moreover, it is remarkable that $U(b, Y)$ is selected from the EFT of continuous media at LO in derivatives by the symmetry $V_{s}$ Diff $\times T_{s}$; see Fig. 1 . In addition, thanks to this symmetry, $U(b, Y)$ includes all four Stückelberg fields needed for a full embedding of the fluid in spacetime, but without introducing two different four-vectors; see Sec. IV and also [7]. It is also worth pointing out that $U(b, Y)$ is a nonbarotropic (and nonisentropic) fluid, which opens the possibility of describing the dynamics and thermodynamics of a broad variety of physical systems. In summary, for a complete thermodynamic description of perfect fluids at low energies as simple systems, the standard pull-back formalism 
must be extended with an extra scalar, $\Phi^{0}$, and with the symmetry $V_{s}$ Diff $\times T_{s}$.

\section{THERMODYNAMICS WITH BROKEN SHIFT SYMMETRY?}

In this section, we argue that the shift symmetries (4.1) are essential for a consistent thermodynamic interpretation of the EFT of perfect fluids. Generically, it is sufficient that the action of the four Stückelberg fields does not respect one of these symmetries to prevent a proper thermodynamic description. To illustrate this point, we will consider the simplest possible example, assuming that the shift symmetry (4.1) of the case $U(X)$ is broken by the explicit appearance of $\Phi^{0}$ on the master function. So, we consider a k-essence Lagrangian [37-40], given by $U\left(X, \Phi^{0}\right)$. The (nonvanishing) covariant divergence of the current $\mathcal{X}^{\mu}=$ $-2 \sqrt{-X} U_{X} \mathcal{V}^{\mu}$ is the equation of motion for $\Phi^{0}$, i.e. $\nabla^{\mu} \mathcal{X}_{\mu}=U^{\prime}$, where $U^{\prime}=\partial_{\Phi^{0}} U$. The EMT is formally of the same form as for $U(X)$, thus describing a perfect fluid with $\rho=2 X U_{X}-U, p=U$ and four-velocity $\mathcal{V}_{\mu}$. From the relations (3.4) and (3.5), we find

$$
s_{\nu} \nabla^{\nu} T+n_{\nu} \nabla^{\nu} \mu=\sqrt{-X} U^{\prime}+\mathcal{X}^{\mu} \nabla_{\mu} \sqrt{-X} .
$$

Let us now attempt to construct a thermodynamic interpretation of k-essence by considering the various possible combinations of two independent variables among $n, T, \mu$ and $s$, in the same way as in the previous section:

(i) $(\mu, s)$ : In this case we get $X=-\mu^{2}$ and $\Phi^{0}$ is independent from $\mu$ and $s$. We find that $T=0$ and $\mathcal{X}^{\mu}=n u^{\mu}$, so that (6.1) implies that $U^{\prime}=0$.

(ii) $(n, T)$ : Then $X=-T^{2}$ and $\Phi^{0}$ is independent from $n$ and $T$. Besides, $\mu=0$ and $\mathcal{X}^{\mu}=s u^{\mu}$. Again, (6.1) implies that $U^{\prime}=0$.

(iii) $(\mu, T)$ : Taking $U(X(\mu, T)=p(\mu, T)$, from the Euler and the Gibbs-Duhem relations, we obtain that $X=-\mu^{2} f_{1}(T / \mu)$ and $\phi=f_{2}(T / \mu)$ with $s=-f_{1}^{\prime} U_{X} \sqrt{-X / f_{1}}+f_{2}^{\prime} U^{\prime} / \sqrt{-X / f_{1}}$ and $n=$ $\left(f_{1}^{\prime} T-2 \sqrt{-X f_{1}}\right) U_{X}+f_{2}^{\prime} f_{1} T U^{\prime} / X$. Since the correspondence must be valid for all $U\left(X, \Phi^{0}\right)$, the equation (6.1) implies that $U^{\prime}=0$.

The thermodynamic relations are incompatible with the equations of motion unless the Lagrangian does not depend on $\Phi^{0}$. We conclude that $k$-essence only admits a thermodynamic interpretation if the shift symmetry is enforced, that is: if the action depends only on $X$. The above statement can be easily extended to the other cases.

\section{SOME SIMPLE APPLICATIONS OF THE THERMODYNAMIC DICTIONARY}

\section{A. Bose-Einstein and Fermi-Dirac distributions}

Consider the Bose-Einstein (BE) and Fermi-Dirac (FD) statistics for (noninteracting) particles of mass $m$ with spin degeneracy $g$ per energy state. The number of particles per unit of momentum $k$ at temperature $T$ is a function of the chemical potential $\mu$ :

$$
2 \pi^{2} F(k, T, \mu)=\frac{g}{\exp \left[\left(\sqrt{k^{2}+m^{2}}-\mu\right) / T\right]+\epsilon}, \quad \epsilon= \pm 1 .
$$

In this expression, $\epsilon=1$ corresponds to FD and $\epsilon=-1$ to BE. For convenience, we have set the Boltzmann constant to be 1 . Some interesting limits are controlled by the ratios $m / T$ and $z=\mu / T$. If $z \gg 1$, the gas becomes degenerate; whereas, if $z \ll 1$ the particles have more freedom to occupy higher energy levels. The deeply relativistic limit is $T \gg m$ and, conversely, the gas becomes nonrelativistic at sufficiently low temperatures, i.e. $m \gg T$.

For $T \gg m$, we get

$$
\begin{aligned}
& p=\frac{\rho}{3}=-\epsilon \frac{g}{\pi^{2}} T^{4} \mathrm{Li}_{4}(-\epsilon \alpha), \quad n=-\epsilon \frac{g}{\pi^{2}} T^{3} \mathrm{Li}_{3}(-\epsilon \alpha), \\
& s=\frac{4}{3} \frac{\rho}{T}-n \log (\alpha),
\end{aligned}
$$

where $\operatorname{Li}_{x}(z)$ is the polylogarithm function of order $x$ [41] and $\alpha=\exp (z)$ is the fugacity. Using Table II, we can reproduce these relations by choosing $(\mu, T)$ and taking

$U(Y)=\frac{g}{3} Y^{4}, \quad Y=T f(z)=T\left[-\frac{\epsilon}{\pi^{2}} \mathrm{Li}_{4}\left(-\epsilon e^{z}\right)\right]^{1 / 4}$.

The high temperature limit of a deeply relativistic gas is obtained taking $\alpha \rightarrow 0$, which gives $Y \propto T$. In the lowtemperature limit, $\alpha \rightarrow \infty$, the gas is degenerate and $\mathrm{Li}_{n}(-\epsilon \alpha) \rightarrow \frac{\alpha^{n}}{n \epsilon}$. As a result, $Y \propto \mu$. The same conclusions can be reached by using instead $U=g X^{2} / 3$, with $X=$ $T^{2} f_{2}(\alpha)$ and $f_{2}(\alpha)=\left[\frac{-\epsilon}{\pi^{2}} \operatorname{Li}_{4}(-\epsilon \alpha)\right]^{1 / 2}$.

In the nonrelativistic limit $m \gg T$, the expressions (7.1) become

$n=g\left(\frac{m T}{2 \pi}\right)^{3 / 2} \exp \left(\frac{\mu-m}{T}\right), \quad \rho=n\left(m+\frac{3 T}{2}\right)$,

$p=n T \ll \rho$.

These can be reproduced with $U(b, Y)$, where $b=n$, $Y=T$, and taking

$$
U(b, Y)=b Y\left\{1+\log \left[\frac{g}{b}\left(\frac{m Y}{2 \pi}\right)^{3 / 2}\right]\right\}-b m .
$$

When both $\alpha$ and $m / T$ are non-negligible, getting a relation among the EFT operators $b$ and $Y$ and the thermodynamic variables involves solving an 
integrodifferential equation for $U(b, Y)$, using $b=n(T, \mu)$ and $\mu=-U_{b}+Y U_{b Y}$.

\section{B. van der Waals gas}

In this case the pressure and the particle number density are related by $\left(p+\kappa n^{2}\right)(1-\gamma n)=T n$, where the constants $\kappa$ and $\gamma$ model the finite molecular volume of the gas and small intermolecular interactions, respectively. This equation of state can be easily obtained from $U(b, Y)$ choosing the pair $\{n, T\}$ as independent thermodynamic variables, so $b=n$ and $Y=T$. Integrating $p=U-b U_{b}$ we obtain $U=b\left[b \kappa+Y \log \left(b^{-1}-\gamma\right)+\mathcal{U}(Y)\right]$. The function $\mathcal{U}(Y)$ can be determined imposing that for $\kappa=0$ and $\gamma=0$ we recover the expression (7.5) for the ideal gas, which leads to

$U(b, Y)=b\left\{b \kappa+Y-m+Y \log \left[\frac{g(1-\gamma b)}{b}\left(\frac{m Y}{2 \pi}\right)^{3 / 2}\right]\right\}$,

and hence

$\rho=b\left(m+\frac{3}{2} T-n \kappa\right)$,

$s=\frac{5 b}{2}+b \log \left[\left(\frac{m Y}{2 \pi}\right)^{3 / 2} \frac{g(1-b \gamma)}{b}\right]$,

$\mu=m-2 b \kappa+\frac{b Y \gamma}{1-b \gamma}+Y \log \left[\frac{b}{g(1-b \gamma)}\left(\frac{m Y}{2 \pi}\right)^{-3 / 2}\right]$.

\section{Polytropic fluids}

Polytropic fluids are used to model the behavior of matter under a wide range of physical conditions, including, e.g., the interior of neutron stars. It is convenient to separate the mass contribution $\rho_{0}=n m$ ( $m$ is the individual mass of the fluid's constituents) and the internal energy density $\epsilon_{I}$ from the energy density $\rho=\rho_{0}+\epsilon_{I}$. The equation of state then reads $p=\kappa \rho_{0}^{\Gamma}$, with $\Gamma$ constant. A polytropic equation of state can be described by $U(b)=\lambda b+\kappa \frac{b^{\Gamma}}{1-\Gamma}$, taking $\{n, T\}$ as thermodynamic variables. In this case, $b=n$ and $\rho_{0}=b m, p(b)=$ $U-b U_{b}=\kappa b^{\Gamma}$. Polytropic equations of state can also be described by $U(Y)$ and $U(X)$, though the expressions are more involved and will be omitted.

\section{Ehrenfest-Tolman effect}

If the spacetime curvature is nonzero and there exists a timelike Killing vector $\xi^{\mu}$, the equilibrium temperature $T$ satisfies-according to the Ehrenfest-Tolman effect- the relation
$T \sqrt{-g_{\mu \nu} \xi^{\mu} \xi^{\nu}}=$ constant.

An analogous relation holds as well for the chemical potential $\mu$; see for instance [42]. The temperature $T$ and the chemical potential $\mu$ of our dictionary-see Table IIare consistent with the Ehrenfest-Tolman result provided that $v^{\mu} \propto \xi^{\mu}$ and $\xi^{\mu} \partial_{\mu} \Phi^{0}=1$. Indeed, $T$ and $\mu$ can be associated to $Y$ and $-X^{2}$, respectively. For a static spacetime, the timelike Killing vector $\xi^{\mu}$ can be identified with $\partial^{\mu} \Phi^{0}$ and thus (7.8) holds, showing that the EhrenfestTolman effect takes place.

\section{THERMODYNAMIC STABILITY}

Thermodynamic stability of a system requires that the Hessian matrix of the function $s(\rho, n)$ must be negative definite, ${ }^{6}$ which is guaranteed by the conditions

$$
s_{\rho \rho}+s_{n n} \leq 0 \quad \text { and } \quad s_{\rho \rho} s_{n n}-s_{\rho n}^{2} \geq 0 .
$$

These equations can also be equivalently formulated in terms of conditions for the energy density, whose Hessian must be positive definite:

$$
\rho_{s s}+\rho_{n n} \geq 0 \quad \text { and } \quad \rho_{s s} \rho_{n n}-\rho_{s n}^{2} \geq 0 .
$$

It is also possible to use the other potentials of Table I. In particular, for the free-energy density $\mathcal{F}(n, T)=\rho-T s$, the conditions reduce simply to $\mathcal{F}_{n n} \geq 0$ and $\mathcal{F}_{T T} \leq 0$ (while the condition on the mixed second derivative is automatically implied by these two). Similarly, for the potential $\mathcal{I}(\mu, s)=\rho-\mu n$ the conditions are $\mathcal{I}_{\mu \mu} \leq 0$ and $\mathcal{I}_{s s} \geq 0$. If any of these potentials $(\mathcal{F}$ or $\mathcal{I})$ are identified with $-U$, which according to Table II occurs only for $U(b, Y)$, the thermodynamic stability conditions are translated into simple constraints on the derivatives of $U$. Concretely:

$$
U_{b b} \leq 0 \quad \text { and } \quad U_{Y Y} \geq 0 .
$$

Similarly, for the grand potential $\omega(\mu, T)=-p=$ $\rho-s T-n \mu$, thermodynamic stability is guaranteed when

$$
\omega_{\mu \mu}+\omega_{T T} \leq 0, \quad \omega_{\mu \mu} \omega_{T T}-\omega_{\mu T}^{2} \geq 0 .
$$

If $-U$ is identified with $\rho$ or $\omega$, as it is the case for the barotropic fluids that depend only on $X, b$ or $Y$, the thermodynamic dictionary involves an undetermined function, $f$, of a ratio of independent thermodynamic variables; see Table II. In these cases, thermodynamic stability leads not only to conditions on the derivatives of $U$, but also to

\footnotetext{
${ }^{6} \mathrm{~A} 2 \times 2$ matrix $M$ is negative definite if $\operatorname{Tr}(M) \leq 0$ and $\operatorname{det}(M) \geq 0$. Conversely, it is positive definite if $\operatorname{Tr}(M) \geq 0$ and $\operatorname{det}(M) \geq 0$.
} 
some constraints involving derivatives of $f$. Concretely, for $\rho=-U(b)$ we get

$$
U_{b b} \leq 0 \text { and } f^{\prime \prime} U_{b} \leq 0,
$$

where primes denote derivatives of $f$ with respect to its argument; see Table II. Similarly, for $\omega=-U(Y)$ we obtain

$$
U_{Y Y} \geq 0 \text { and } f^{\prime \prime} U_{Y} \geq 0 .
$$

Finally, in the case $\omega=-U(X)$, we get

$$
2 X U_{X X}+U_{X} \leq 0, \quad \text { and } \quad\left(\left(f^{\prime}\right)^{2}-2 f f^{\prime \prime}\right) U_{X} \geq 0 .
$$

If we take the case of the free relativistic $\mathrm{BE}$ or $\mathrm{FD}$ distribution (see Sec. VII), we can specify the function $f(z)$, where $z=\mu / T$, and then we get: $f^{\prime \prime}(z) \geq 0$ when $f \sim\left(-\epsilon \operatorname{Li}_{4}\left(-\epsilon e^{z}\right)\right)^{1 / 4}$ (which is the case for the $U(Y)$ and it implies $\left.U_{Y} \geq 0\right)$ while $\left(\left(f^{\prime}\right)^{2}-2 f f^{\prime \prime}\right) \leq 0$ when $f \sim$ $\left(-\epsilon \operatorname{Li}_{4}\left(-\epsilon e^{z}\right)\right)^{1 / 2}$ (for the $U(X)$ case and it implies $\left.U_{X} \leq 0\right)^{7}$

\section{SOUND SPEED}

We can define the sound speed of perfect fluids as the quantity that relates the variations of the energy density and the pressure along the fluid flow:

$$
p^{\prime}=c_{s}^{2} \rho^{\prime}
$$

As we will see in the next section, where we discuss dynamical stability, this definition gives the speed of propagation of longitudinal phonons, appearing naturally by expanding the action at quadratic order in fluctuations. For barotropic perfect fluids, (9.1) can be simply computed as $c_{s}^{2}=d p / d \rho$ using the expressions (4.11), giving

$$
c_{s}^{2}= \begin{cases}b \frac{U_{b b}}{U_{b}}, & U=U(b) \\ \frac{U_{Y}}{Y U_{Y Y}}, & U=U(Y) . \\ \frac{U_{X}}{U_{X}+2 X U_{X X}}, & U=U(X)\end{cases}
$$

In the case of $U(b, Y)$, a variation of the pressure is not uniquely determined by the variation of the energy density. This is simply because $U(b, Y)$ is a nonbarotropic fluid; see (4.12). In this case, it is convenient to define the restricted variations of the pressure with respect to the energy density

\footnotetext{
${ }^{7}$ In the $\mathrm{BE}$ case $(\epsilon=1)$, the chemical potential $\mu$ is negative and $0 \leq \alpha \leq 1$.
}

$c_{b}^{2}=\left.\frac{\partial p}{\partial \rho}\right|_{b}=\frac{U_{Y}-b U_{b Y}}{Y U_{Y Y}}, \quad c_{Y}^{2}=\left.\frac{\partial p}{\partial \rho}\right|_{Y}=\frac{b U_{b b}}{U_{b}-Y U_{b Y}}$.

To obtain the relation between the variations of the energy density and the pressure we make use of the conservation of the currents $\mathcal{J}^{\mu}=b u^{\mu}$ and $\mathcal{Y}^{\mu}=U_{Y} u^{\mu}$, defined in (4.8) and (4.10). This can be written as $b^{\prime}+$ $\theta b=0$ and $U_{Y b} b^{\prime}+U_{Y Y} Y^{\prime}+\theta U_{Y}=0$, where $\theta=\nabla_{\mu} u^{\mu}$ is the expansion, that we introduced in Sec. III. Combining these two equations to eliminate $\theta$, we get

$$
b Y^{\prime}=c_{b}^{2} Y b^{\prime}
$$

Using this result, the sound speed (9.1) can be easily computed as (see also [16])

$$
c_{s}^{2}=\frac{p_{b} b^{\prime}+p_{Y} Y^{\prime}}{\rho_{b} b^{\prime}+\rho_{Y} Y^{\prime}}=\frac{c_{b}^{4} Y \rho_{Y}+b p_{b}}{\rho+p},
$$

where, as we have been doing through, the subscripts in $U, \rho$ and $p$ denote partial derivatives, e.g.

$$
\rho_{Y}=\left.\frac{\partial \rho}{\partial Y}\right|_{b}=Y U_{Y Y}, \quad p_{b}=\left.\frac{\partial p}{\partial b}\right|_{Y}=-b U_{b b} .
$$

Unsurprisingly, (9.5) reduces to one of the expressions (9.2) if $b$ or $Y$ are absent from the action. The quantities (9.4), (9.5), and (9.6) allow to express generic variations of the pressure and energy density as follows,

$\delta p=p_{b} \delta b+c_{b}^{2} \rho_{Y} \delta Y, \quad \delta \rho=\rho_{Y} \delta Y+c_{Y}^{-2} p_{b} \delta b$,

so clearly, $\delta p \neq c_{s}^{2} \delta \rho$. The missing ingredient that allows to turn this into an equality is the variation of the entropy density per particle, $\sigma=s / n$, which we can compute using the dictionary of Table II, obtaining

$$
\begin{aligned}
\delta \sigma & =\alpha \frac{\rho_{Y}}{b}\left(\frac{\delta Y}{Y}-c_{b}^{2} \frac{\delta b}{b}\right), \\
\alpha & = \begin{cases}-\left(b / U_{Y}\right)^{2}=-\sigma^{2}, & \text { for }\{\mu, s\} \\
1, & \text { for }\{n, T\}\end{cases}
\end{aligned}
$$

so that

$$
\delta p=c_{s}^{2} \delta \rho+\frac{b Y}{\alpha}\left(c_{b}^{2}-c_{s}^{2}\right) \delta \sigma .
$$

We recall that in a perfect fluid-for which the entropy and particle currents are parallel-if one of them is conserved the other is also conserved, which implies that $\sigma^{\prime}=0$; see Sec. III. This is precisely what happens in the case at hand, where $\mathcal{Y}^{\mu}$ and $\mathcal{J}^{\mu}$ are aligned with $u^{\mu}$; see Table II for their interpretation according to the choice of 
thermodynamic variables. Notice also that $\sigma^{\prime}=0$ does not imply in general that $\sigma$ is vanishing. It is important to emphasize that these results rely on the thermodynamic interpretation of the EFT action, which allows us to identify the currents of entropy and density. Clearly, the thermodynamic interpretation is also needed to compute $\sigma$ and its variation (9.8). It is also worth stressing that these arguments are valid at all orders in fluctuations, as it is clear from the way we have constructed the variations in (9.8).

\section{PROPAGATION OF PHONONS AND DYNAMICAL STABILITY}

In this section, we study the propagation of the internal degrees of freedom of effective perfect fluids and their relation to energy and pressure perturbations, as well as the conditions that ensure dynamical stability. The results of this analysis will be related to thermodynamic stability, which was discussed in Sec. VIII. For simplicity, we will consider perfect fluids living in flat spacetime. Then, the dynamics of linear fluctuations are given by the Euler and the continuity equations (EE and $\mathrm{CE}$ ), which read, respectively, ${ }^{8}$

$$
\begin{aligned}
& (\rho+p) \partial_{t} v^{i}+\partial_{i} \delta p=0, \\
& \partial_{t} \delta \rho+(\rho+p) \partial_{i} v^{i}=0,
\end{aligned}
$$

and come from the conservation of $T_{\mu \nu}$; see (3.2) and (3.4). To solve them, an extra relation between $\delta p, \delta \rho$ and $\partial_{i} v^{i}$ needs to be known, which in our case is provided by the EFT action. The expansion at the second order in the phonon fields of the three operators of the EFT is given by

$b=1+\partial_{i} \pi^{i}-\frac{1}{2} \dot{\pi}^{i} \dot{\pi}^{i}+\frac{1}{2}\left(\partial_{i} \pi^{i}\right)^{2}-\frac{1}{2}\left(\partial_{i} \pi^{j}\right)\left(\partial_{j} \pi^{i}\right) ;$

$$
\begin{gathered}
Y=1+\dot{\pi}^{0}+\frac{1}{2} \dot{\pi}^{i} \dot{\pi}^{i}-\dot{\pi}^{i} \partial_{i} \pi^{0} \\
X=-1-2 \dot{\pi}^{0}-\left(\dot{\pi}^{0}\right)^{2}-\left(\partial_{i} \dot{\pi}^{0}\right)^{2} .
\end{gathered}
$$

(i) Let us start with $U(b)$ and write the scalar fields as

$$
\Phi^{i}\left(t, x^{j}\right)=x^{i}+\pi^{i}(t, \vec{x}) .
$$

In this expression, $\pi^{i}$ represent the phonons that are fluctuations around the static background $\Phi^{i}=x^{i}$ under the assumption that $\left|\partial_{i} \pi^{j}\right| \ll 1$. Indeed, the

\footnotetext{
${ }^{8}$ In this section, both $\partial_{t}$ and the overdots indicate time derivatives. The background enthalpy per unit of volume, denoted here by $\rho+p$, is a constant in Minkowski spacetime.
}

effective theory of fluids that we are discussing can be seen as the theory of the propagation of sound waves in continuous media [3]. Notice that the fields $\pi^{i}=\Phi^{i}-x^{i}$ are invariant under the combination of a constant translation $x^{i} \rightarrow x^{i}-c^{i}$ and an internal shift $\Phi^{i} \rightarrow \Phi^{i}+c^{i}$. Therefore, the $\pi^{i}$ propagate on a homogeneous background and represent the Goldstone bosons of broken translations. It is convenient to split the phonons into a longitudinal component and two transverse ones, defined by

$$
\pi^{i}=\pi_{L}^{i}+\pi_{T}^{i}, \quad \pi_{L}^{i}=\partial_{i} \pi_{L}, \quad \partial_{i} \pi_{T}^{i}=0 .
$$

The action expanded at quadratic order reads

$$
\begin{aligned}
S^{(2)}[b]= & \frac{1}{2}(\rho+p) \\
& \times \int d^{4} x\left[\dot{\pi}_{T}^{i} \dot{\pi}_{T}^{i}-\dot{\pi}_{L} \Delta \dot{\pi}_{L}-c_{s}^{2}\left(\Delta \pi_{L}\right)^{2}\right],
\end{aligned}
$$

where $\Delta=\partial_{i} \partial_{i}$ and $c_{s}^{2}$ can be read in (9.2). The equations of motion (EOMs) are $\ddot{\pi}_{T}^{i}=0$ and $\ddot{\pi}_{L}-c_{s}^{2} \Delta \pi_{L}=0$. Therefore, the transverse modes do not propagate - their amplitude simply changes linearly in time-due to the conservation of vorticity, which can be traced back to the symmetry $V_{s}$ Diff. The longitudinal mode propagates with speed of sound given by (9.2). The linear pressure and density perturbations are $\delta p=c_{s}^{2} \delta \rho=$ $c_{s}^{2}(\rho+p) \Delta \pi_{L}$, and indeed $c_{s}^{2}=d p / d \rho$, as can be seen directly from (4.11). The linear velocity perturbation is $u^{i}=-\dot{\pi}^{i}$, and the divergence of the EE (10.1) is nothing but the equation for the propagation of $\pi_{L}$. The CE (10.2) is simply an identity. Stability requires $\rho+p \geq 0$, in order to avoid possibly dangerous ghosts, and $c_{s}^{2} \geq 0$, to avoid exponential growth of $\pi_{L}$. The first condition is $U_{b} \leq 0$. If this is satisfied, the constraint $c_{s}^{2} \geq 0$ is equivalent to the condition for thermodynamic stability $U_{b b} \geq 0$ of (8.3).

(ii) Let us now consider the irrotational perfect fluid $U(X)$. The only scalar that is present in this case is $\Phi^{0}$, which we write in a similar fashion as (10.6): $\Phi^{0}=\varphi(t)+\pi^{0}$. The EOM for $\varphi$ is $\partial_{t}\left(U_{x} \dot{\varphi}\right)=0$ and implies that $\varphi \propto t$ (except if $\rho \rightarrow 0$, which is a limit we discard). So, we write

$$
\Phi^{0}=t+\pi^{0},
$$

and expand the action assuming that $\left|\partial \pi^{0}\right| \ll 1$. The dynamics of the Goldstone boson of the broken time translation is then governed by the action 
$S^{(2)}[X]=\frac{1}{2}(\rho+p) \int d^{4} x\left[c_{s}^{-2}\left(\dot{\pi}^{0}\right)^{2}-\left(\partial_{i} \pi^{0}\right)^{2}\right]$,

where $c_{s}^{2}$ is given in (9.2). The linear pressure and energy density perturbations are $\delta p=-2 p_{X} \dot{\pi}^{0}=$ $c_{s}^{2} \delta \rho$ and, again, $c_{s}^{2}=d p / d \rho=p_{X} / \rho_{X}$ from (4.11). The velocity perturbation is $\mathcal{V}^{i}=-\partial_{i} \pi^{0}$. The EOM for $\pi^{0}$ is $\ddot{\pi}^{0}-c_{s}^{2} \Delta \pi^{0}=0$, which is the CE (10.2), whereas the EE (10.1) is now an identity; just the opposite of what occurs for $U(b)$. Dynamical stability requires $(\rho+p) / c_{s}^{2} \geq 0$ and $\rho+p \geq 0$. Thermodynamic stability, see (8.7), demands precisely the first of these two conditions.

(iii) We will now consider $U(Y)$, which contains both types of scalar fields, $\Phi^{i}$ and $\Phi^{0}$. The appropriate background in this case is given by (10.6) and (10.9), as it can be checked using the EOMs. In particular, the conservation of (4.10) implies that $U_{Y}$ is a constant, which then requires $\Phi^{0} \propto t$. The quadratic action for the phonons around the background is

$$
\begin{aligned}
S^{(2)}[Y]= & \frac{1}{2}(\rho+p) \int d^{4} x\left[\dot{\pi}_{T}^{i} \dot{\pi}_{T}^{i}-\dot{\pi}_{L} \Delta \dot{\pi}_{L}\right. \\
& \left.-c_{s}^{-2}\left(\dot{\pi}^{0}\right)^{2}-2 \dot{\pi}^{0} \Delta \pi_{L}\right],
\end{aligned}
$$

where, once more, $c_{s}^{2}$ is given in (9.2). The EOMs derived from the above action, besides $\ddot{\pi}_{T}^{i}=0$, are

$$
\ddot{\pi}_{L}-\dot{\pi}^{0}=0, \quad \ddot{\pi}^{0}-c_{s}^{2} \Delta \dot{\pi}_{L}=0 .
$$

As in the case $U(b), \ddot{\pi}_{T}^{i}=0$ is a consequence of the symmetry $V_{s}$ Diff.

Equating to zero the determinant of the quadratic form that defines the Lagrangian of (10.11) and going to Fourier space, one finds two modes: one with a dispersion relation $\omega^{2}-c_{s}^{2} k^{2}=0$ and a second one with $\omega=0$. This second mode is similar to the transverse ones, $\pi_{T}^{i}$, which also have $\omega=0$. Notice, as well, that once the equation of motion for $\pi_{L}$ is solved, the dynamics of $\pi^{0}$ is given by a single time integration constant.

As in the case $U(X)$, the energy density and pressure perturbations depend on $\dot{\pi}^{0}$, i.e. $\delta p=$ $c_{s}^{2} \delta \rho=c_{s}^{2}(\rho+p) \dot{\pi}^{0}$, whereas the velocity perturbation is the same as for $U(b)$, i.e. $u^{i}=-\dot{\pi}^{i}$. The $\mathrm{EE}$ is the first of the EOMs derived from (10.11) and the CE (10.2) is the other one, since $u^{i}$ depends on $\pi^{i}$ while $\delta \rho$ depends on $\pi^{0}$. In this case dynamical stability requires $\rho+p>0$ and $c_{s}^{2}>0$ as it can be checked by imposing that the Hamiltonian density derived from (10.11) is positive. When
$\rho+p=U_{Y}>0$ holds, thermodynamic stability implies that $c_{s}^{2}>0$; see (8.3).

To summarize the results so far, we have seen that for perfect fluids $U(b), U(X)$, and $U(Y)$ longitudinal modes - a single mode for $U(b)$ and $U(X)$ and two for $U(Y)$ - propagate with speeds of sound $c_{s}^{2}=d p / d \rho$, given in (9.2). This reflects that these fluids are barotropic. We have seen that in these three cases, thermodynamic stability plus the nullenergy condition imply positivity of the speed of sound, ensuring dynamical stability.

(iv) Finally, let us focus on $U(b, Y)$. Expanding as before around $\Phi^{i}=x^{i}$ and $\Phi^{0}=t$, we obtain

$$
\begin{aligned}
S^{(2)}[b, Y]= & \frac{1}{2} \int d^{4} x\left[(\rho+p)\left(\dot{\pi}_{T}^{i} \dot{\pi}_{T}^{i}-\dot{\pi}_{L} \Delta \dot{\pi}_{L}\right)\right. \\
& \left.+\rho_{Y}\left(\dot{\pi}^{0}\right)^{2}-p_{b}\left(\Delta \pi_{L}\right)^{2}-2 c_{b}^{2} \rho_{Y} \dot{\pi}^{0} \Delta \pi_{L}\right] .
\end{aligned}
$$

As before, $V_{s}$ Diff gives $\ddot{\pi}_{T}^{i}=0$, and the remaining EOMs are

$$
\begin{aligned}
\ddot{\pi}^{0}-c_{b}^{2} \Delta \dot{\pi}_{L} & =0, \\
(\rho+p) \ddot{\pi}_{L}-\rho_{Y} c_{b}^{2} \dot{\pi}^{0}-p_{b} \Delta \pi_{L} & =0,
\end{aligned}
$$

which give

$$
\begin{aligned}
\dot{\pi}^{0} & =c_{b}^{2} \Delta \pi_{L}+\sigma_{0}(\vec{x}), \\
\ddot{\pi}_{L}-c_{s}^{2} \Delta \pi_{L} & =\frac{\rho_{Y} c_{b}^{2}}{\rho+p} \sigma_{0}(\vec{x}),
\end{aligned}
$$

where $\sigma_{0}(\vec{x})$ is a generic time-independent function, fixed by initial conditions. The situation is analogous to the case $U(Y)$ with two modes, one that propagates with velocity $c_{s}^{2}$ given by (9.5), and a second one with $\omega=0$. The reason is the lack of a quadratic term in (10.13) containing only spatial derivatives of $\pi^{0}$.

The Hamiltonian density $\mathcal{H}$ derived from (10.13) is given by $\mathcal{H}=P_{0} \dot{\pi}^{0}+P_{L} \dot{\pi}_{L}+\vec{P} \cdot \dot{\vec{\pi}}_{T}-\mathcal{L}$, where $\mathcal{L}$ is the Lagrangian density and $P_{0}, P_{L}, \vec{P}$ are the momenta conjugate to $\pi^{0}, \pi_{L}$, and $\vec{\pi}_{T}$, respectively. As a result,

$$
\begin{aligned}
\mathcal{H}= & \frac{1}{2}\left[(p+\rho)\left(\dot{\vec{\pi}}_{T} \cdot \dot{\vec{\pi}}_{T}+\vec{\nabla} \dot{\pi}_{L} \cdot \vec{\nabla} \dot{\pi}_{L}\right)\right. \\
& \left.+U_{Y Y}\left(\dot{\pi}^{0}\right)^{2}-U_{b b}\left(\Delta \pi_{L}\right)^{2}\right] .
\end{aligned}
$$

Thus, $\mathcal{H}$ is positive definite when $\rho+p=U_{Y}-U_{b}>0$, $U_{b b}<0$ and $U_{Y Y}>0$. Notice that the previous three conditions also ensure that $c_{s}^{2}>0$; see (9.5). The entropy perturbation is nonvanishing. Indeed, at linear order, by using the dictionary entry for $U(b, Y)$ relative to $(n, T)$, we have that 


$$
\delta \sigma \equiv \rho_{Y}\left(\dot{\pi}^{0}-c_{b}^{2} \Delta \pi_{L}\right)=\rho_{Y} \sigma_{0}(\vec{x}) .
$$

It is straightforward to see from (10.15) that although $\delta \sigma \neq 0$, it is constant in time,

$$
\partial_{t}(\delta \sigma)=0,
$$

as expected for an adiabatic fluid. The conclusion is the same when the dictionary entry for $U(b, Y)$ relative to $(\mu, s)$ is used. The analysis of Sec. VIII showed that thermodynamic stability requires $U_{b b} \leq 0$ and $U_{Y Y} \geq 0$. As in the previous cases, thermodynamic stability plus the nullenergy condition imply dynamical stability. Notice that in the cases where an unspecified function appears in the dictionary, thermodynamic stability implies additional constraints on $f$ that cannot be obtained requiring dynamical stability of the phonons. However, in the examples where $f$ is known, such as those of Sec. VII, those additional constraints are automatically satisfied.

\section{SUMMARY AND CONCLUSIONS}

We have studied thermodynamic interpretation of the EFT of perfect fluids, obtaining a dictionary summarized in Table II, completing and extending the results of $[4,5]$. We have established the correspondence between the fundamental thermodynamic variables needed to describe simple systems and the EFT operators that configure the four types of perfect fluids that are allowed in the theory. Each entry of the dictionary (described by Table II) corresponds to a specific operator content in the EFT. The interpretation of the EFT master function $U$ - the Lagrangian - as a thermodynamic potential is determined by the EMT. For the effective perfect fluids depending on a single scalar operator, the master function $U$ represents either the energy density (for $U(b)$ ) or the pressure (for $U(Y)$ and $U(X)$ ). For these cases, the thermodynamic potentials $\mathcal{F}$ and $\mathcal{I}$ appears as specific limits of the free function $f$ of Table II. For the Lagrangian $U(b, Y)$, the master function corresponds to a Legendre transformation of the energy density (or the pressure) to another thermodynamic potential, such as the free energy $\mathcal{F}=\rho-T s$ or to the potential $\mathcal{I}=\rho-\mu n$.

A full thermodynamic correspondence, allowing to identify simultaneously an independent and conserved particle (or charge) number current and a conserved entropy density current is only possible for perfect fluids described by the Lagrangian $U(b, Y)$. This action is invariant under the internal symmetry group $V_{s}$ Diff $\times T_{s}$ of spatial volume preserving diffeomorphisms and time redefinitions that depend on the spatial fields. The fact that $U(b, Y)$ is the only perfect fluid Lagrangian that is chosen by a (continuous) symmetry (assuming four scalars), highlights it even more as the most complete effective description of perfect fluids; see also [5]. The other perfect fluids, indicated as $U(b), U(Y)$ and $U(X)$ in Table II, can also be given thermodynamic interpretations, but only in limits where a single thermodynamic variable is sufficient for the description. Clearly, this is because two independent operators ( $b$ and $Y$ ) are needed for a nondegenerate matching with two independent thermodynamic variables; and also to describe two independent thermodynamic conserved currents. We have illustrated the use of the thermodynamic dictionary with a few well-known cases of perfect fluids, such as e.g. Bose-Einstein and Fermi-Dirac gases in the deeply relativistic limit and a van der Waals gas.

We have argued that internal shift symmetries are a necessary condition for a thermodynamic description by studying the Lagrangian for a single scalar with an explicitly broken shift symmetry: $U(X, \phi)$, whose EMT has the form of a perfect fluid, showing that in such a case the basic thermodynamic relations are incompatible with the equation of motion of the field.

We have also studied the propagation of linearized sound waves in flat spacetime and how the Euler and the continuity equations describe the dynamics of Goldstone bosons for each kind of perfect fluid. This analysis leads to the conclusion that thermodynamic stability plus the nullenergy condition, $\rho+p \geq 0$, ensure dynamical stability. This holds true for the four possible types of effective perfect fluids. The same analysis shows that the fluid described by $U(b, Y)$, being in general nonbarotropic, can support nonvanishing entropy per particle perturbations but is nonetheless adiabatic.

\section{ACKNOWLEDGMENTS}

We thank B. Bellazzini, L. Hui, I. Sawicki, and S. Sibiryakov for useful discussions. L. P. thanks S. Ciuchi for interesting discussions and suggestions. The work of G. B. is funded by the European Union's Horizon 2020 research and innovation program under the Marie SkłodowskaCurie Grant Agreement No. 656794. G. B. thanks the CERN Theoretical Physics Department for hospitality while this work was done. L. P. thanks the Institute de Physique Théorique IPhT CEA-Saclay for hospitality. We also thank the Galileo Galilei Institute for Theoretical Physics for hospitality and the Instituto Nazionale di Fisica Nucleare (INFN) for support during the completion of this work.

\section{APPENDIX: A SYSTEMATIC APPROACH TO THE THERMODYNAMIC DICTIONARY}

Any simple thermodynamic system is described by at most six variables: $\zeta=\{s, T, n, \mu, \rho, p\}$, of which only two are independent. For the EFT of perfect fluids, $\rho$ and $p$ are given as functions of at most two operators among $\mathcal{O}=\{b, Y, X\}$. Let us choose two independent variables (other than $\rho$ and $p$ ) from the list $\zeta$ and denote them $z_{1}$ and $z_{2}$. These can be, for instance, $\{n, s\}$ or $\{\mu, T\}$; see Table I. Since all the other four variables contained in $\zeta$ are, by assumption, functions of $z_{1}$ and $z_{2}$, we can formally write 


$$
\begin{aligned}
\zeta\left(z_{1}, z_{2}\right)= & \underbrace{\left\{z_{1}, z_{2}\right\}}_{\text {Indep var }} \times \underbrace{\left\{\mathcal{Z}_{1}\left(z_{1}, z_{2}\right), \mathcal{Z}_{2}\left(z_{1}, z_{2}\right)\right\}}_{\text {Dependent variables }} \\
& \times \underbrace{\left\{\rho\left[\mathcal{O}\left(z_{1}, z_{2}\right)\right], p\left[\mathcal{O}\left(z_{1}, z_{2}\right)\right]\right\}}_{\text {Variables from the EMT }},
\end{aligned}
$$

where we have explicitly taken into account that the operators $\mathcal{O}$ must be functions of $z_{1}$ and $z_{2}$, and we have denoted by $\mathcal{Z}_{1}$ and $\mathcal{Z}_{2}$ the two (dependent) variables that are not $\rho$ and $p$. The thermodynamic relations (2.3), (2.5), and (2.7) constrain the dependence of $\mathcal{Z}_{1,2}\left(z_{1}, z_{2}\right)$ and $\mathcal{O}\left(z_{1}, z_{2}\right)$ on the chosen independent variables $z_{1}$ and $z_{2}$. The thermodynamic dictionary for the EFT of perfect fluids can be derived, requiring that

(i) It has to be valid for any master function $U$.

(ii) The operators $\mathcal{O}$ are independent of $U$ and its derivatives.

Then, any thermodynamic constraint involving the derivatives $U^{(n)}$ must hold irrespectively of the form of $U^{(n)}$. Moreover, from the Euler relation (2.5), we see that the dependent variables $\mathcal{Z}_{1,2}\left(z_{1}, z_{2}\right)$ will depend on first derivatives of $U$. One can easily keep track of the number of derivatives entering in each quantity using a counting parameter $\epsilon$, such that

$$
\begin{aligned}
U^{(n)}(\mathcal{O}) & \rightarrow \epsilon^{n} U^{(n)}(\mathcal{O}), \\
\mathcal{Z}_{1,2}^{\left(n_{1}, n_{2}\right)}\left(z_{1}, z_{2}\right) & \rightarrow \epsilon^{1+n_{1}+n_{2}} \mathcal{Z}_{1,2}^{\left(n_{1}, n_{2}\right)}\left(z_{1}, z_{2}\right), \\
\mathcal{O}^{\left(n_{1}, n_{2}\right)}\left(z_{1}, z_{2}\right) & \rightarrow \epsilon^{0} \mathcal{O}^{\left(n_{1}, n_{2}\right)}\left(z_{1}, z_{2}\right) .
\end{aligned}
$$

As an example, let us take the case $U(b, Y)$ with $\left\{z_{1}, z_{2}\right\}=$ $\{n, T\}$ as independent variables. From (4.12), we have that $\rho=Y U_{Y}-U$ and $p=U-b U_{b}$. Then, $\left\{\mathcal{Z}_{1}, \mathcal{Z}_{2}\right\}=$ $\{s(n, T), \mu(n, T)\}$ and $\mathcal{O}=(Y, b)$ are also functions of $n$ and $T$. The Euler relation (2.5) reads

$$
\epsilon\left(Y U_{Y}-b U_{b}\right)=\epsilon[T s(n, T)+n \mu(n, T)],
$$

consistently with (A2). Solving for $s(n, T)$ and inserting it in (2.3), or equivalently in (2.7), we get

$$
\begin{aligned}
& T\left\{\epsilon^{2}\left[b\left(-b_{n} U_{b^{2}}-Y_{n} U_{b Y}\right)-n \mu_{n}\right]+\epsilon Y_{n} U_{Y}\right\} d n \\
& \quad+\left\{\epsilon^{2} T\left[b\left(-b_{T} U_{b^{2}}-Y_{T} U_{b Y}\right)-n \mu_{T}\right]+\epsilon\left(b U_{b}+n \mu-Y U_{Y}+T Y_{T} U_{Y}\right)\right\} d T=0 .
\end{aligned}
$$

The coefficients of $d n$ and $d T$ must vanish independently, for all $U$ and order by order in $\epsilon$. At order $\epsilon$, we get $\epsilon Y_{n}=0$ and $\epsilon\left(b U_{b}+n \mu-Y U_{Y}+T Y_{T} U_{Y}\right)=0$. Differentiating these equations with respect to $n$ and $T$, we obtain $\epsilon^{2} Y_{n n}=\epsilon^{2} Y_{n T}=0$ and

$$
\begin{aligned}
\epsilon^{2} \mu_{n} n^{2} & =\epsilon^{2} n b_{n}\left[\left(Y-T Y_{T}\right) U_{b Y}-b U_{b^{2}}\right]+\epsilon\left[\left(b-n b_{n}\right) U_{b}+\left(T Y_{T}-Y\right) U_{Y}\right], \\
\epsilon^{2} n \mu_{T} & =\epsilon^{2}\left[-b b_{T} U_{b^{2}}+\left(Y b_{T}-\left(T b_{T}+b\right) Y_{T}\right) U_{b Y}+Y_{T}\left(Y-T Y_{T}\right) U_{Y^{2}}\right]-\epsilon\left(b_{T} U_{b}+T Y_{T^{2}} U_{Y}\right) .
\end{aligned}
$$

New terms of order $\epsilon$ appear and they have to vanish, leading to $\left(b-n b_{n}\right) U_{b}+\left(T Y_{T}-Y\right) U_{Y}=0$ and $b_{T} U_{b}+T Y_{T^{2}} U_{Y}=0$. The solution of these last equations (independent from the form of $U$ ) is $b=n b_{n}, Y=T Y_{T}$, $b_{T}=Y_{T T}=0$ and, therefore,

$$
b=n, \quad Y=T, \quad \mu=-U_{b}, \quad s=U_{Y} .
$$

One can now check that all the thermodynamic relations are satisfied at all orders in $\epsilon$. The solution (A5) gives the entry of Table II relative to $U(b, Y)$ with $(n, T)$ as independent variables in our dictionary. All the other entries can be derived in the same way.

[1] N. Andersson and G. L. Comer, Relativistic fluid dynamics: Physics for many different scales., Living Rev. Relativ. 10, 1 (2007).

[2] H. Leutwyler, Nonrelativistic effective Lagrangians, Phys. Rev. D 49, 3033 (1994).

[3] H. Leutwyler, Phonons as goldstone bosons, Helv. Phys. Acta 70, 275 (1997).
[4] S. Dubovsky, T. Gregoire, A. Nicolis, and R. Rattazzi, Null energy condition and superluminal propagation. J. High Energy Phys. 03 (2006) 025.

[5] S. Dubovsky, L. Hui, A. Nicolis, and D. Thanh Son, Effective field theory for hydrodynamics: thermodynamics, and the derivative expansion, Phys. Rev. D 85, 085029 (2012). 
[6] A. Nicolis, R. Penco, F. Piazza, and R. Rattazzi, Zoology of condensed matter: Framids, ordinary stuff, extra-ordinary stuff, J. High Energy Phys. 06 (2015) 155.

[7] G. Ballesteros, D. Comelli, and L. Pilo, Massive and modified gravity as self-gravitating media, arXiv: 1603.02956.

[8] A. Nicolis, R. Penco, and R. A. Rosen, Relativistic fluids, superfluids, solids and supersolids from a coset construction, Phys. Rev. D 89, 045002 (2014).

[9] G. Ballesteros, The effective theory of fluids at NLO and implications for dark energy, J. Cosmol. Astropart. Phys. 03 (2015) 001.

[10] S. Matarrese, On the classical and quantum irrotational motions of a relativistic perfect fluid. 1. Classical theory, Proc. R. Soc. London, Sect. A 401, 53 (1985).

[11] H. Callen, Thermodynamics and an introduction to thermostatistics, 2nd edition (Wiley, New York, 1985).

[12] L. Rezzolla and O. Zanotti, Relativistic Hydrodynamics (Oxford University Press, New York, 2013).

[13] B. Carter, Covariant theory of conductivity in ideal fluid or solid media, Lect. Notes Math., 1385, 1 (1989).

[14] D. T. Son, Effective Lagrangian and Topological Interactions in Supersolids, Phys. Rev. Lett. 94, 175301 (2005).

[15] S. Endlich, A. Nicolis, R. Rattazzi, and J. Wang, The quantum mechanics of perfect fluids, J. High Energy Phys. 04 (2011) 102.

[16] A. Nicolis, Low-energy effective field theory for finitetemperature relativistic superfluids, arXiv:1108.2513.

[17] S. Endlich, A. Nicolis, and J. Wang, Solid inflation, J. Cosmol. Astropart. Phys. 10 (2013) 011.

[18] G. Ballesteros and B. Bellazzini, Effective perfect fluids in cosmology, J. Cosmol. Astropart. Phys. 04 (2013) 001.

[19] J. Bhattacharya, S. Bhattacharyya, and M. Rangamani, Nondissipative hydrodynamics: Effective actions versus entropy current, J. High Energy Phys. 02 (2013) 153.

[20] C. Hoyos, B. Keren-Zur, and Y. Oz, Supersymmetric sound in fluids, J. High Energy Phys. 11 (2012) 152.

[21] G. Ballesteros, B. Bellazzini, and L. Mercolli, The effective field theory of multi-component fluids, J. Cosmol. Astropart. Phys. 05 (2014) 007.

[22] L. V. Delacrétaz, A. Nicolis, R. Penco, and R. A. Rosen, Wess-Zumino Terms for Relativistic Fluids, Superfluids, Solids, and Supersolids, Phys. Rev. Lett. 114, 091601 (2015).

[23] B. Gripaios and D. Sutherland, Quantum Field Theory of Fluids, Phys. Rev. Lett. 114, 071601 (2015).

[24] N. Arkani-Hamed, H. Georgi, and M. D. Schwartz, Effective field theory for massive gravitons and gravity in theory space, Ann. Phys. 305, 96 (2003).
[25] S. L. Dubovsky, Phases of massive gravity, J. High Energy Phys. 10 (2004) 076.

[26] V. A. Rubakov and P. G. Tinyakov, Infrared-modified gravities and massive gravitons, Phys. Usp., 51, 759 (2008).

[27] G. L. Comer and D. Langlois, Hamiltonian formulation for multi-constituent relativistic perfect fluids, Classical Quantum Gravity 10, 2317 (1993).

[28] G. L. Comer and D. Langlois, Hamiltonian formulation for relativistic superfluids, Classical Quantum Gravity 11, 709 (1994).

[29] G. L. Comer, P. Peter, and N. Andersson, Multi-fluid cosmology: An illustration of fundamental principles, Phys. Rev. D 85, 103006 (2012).

[30] D. Blas, M. M. Ivanov, and S. Sibiryakov, Testing Lorentz invariance of dark matter, J. Cosmol. Astropart. Phys. 10 (2012) 057.

[31] A. Pourtsidou, C. Skordis, and E. J. Copeland, Models of dark matter coupled to dark energy, Phys. Rev. D 88, 083505 (2013).

[32] L. Berezhiani and J. Khoury, Dark matter superfluidity and galactic dynamics, Phys. Lett. B 753, 639 (2016).

[33] M. Kopp, C. Skordis, and D. B. Thomas, An extensive investigation of the Generalised dark Matter model, arXiv: 1605.00649.

[34] J. Kang and A. Nicolis, Platonic solids back in the sky: Icosahedral inflation, J. Cosmol. Astropart. Phys. 03 (2016) 050 .

[35] I. M. Khalatnikov and V. V. Lebedev, Relativistic hydrodynamics of a superfluid liquid, Phys. Lett. A 91, 70 (1982).

[36] B. Carter and I. M. Khalatnikov, Equivalence of convective and potential variational derivations of covariant superfluid dynamics, Phys. Rev. D 45, 4536 (1992).

[37] C. Armendariz-Picon, T. Damour, and V. F. Mukhanov, k-inflation, Phys. Lett. B 458, 209 (1999).

[38] C. Armendariz-Picon, V. F. Mukhanov, and P. J. Steinhardt, A Dynamical Solution to the Problem of a Small Cosmological Constant and Late Time Cosmic Acceleration, Phys. Rev. Lett. 85, 4438 (2000).

[39] C. Deffayet, O. Pujolas, I. Sawicki, and A. Vikman, Imperfect dark energy from kinetic gravity braiding, J. Cosmol. Astropart. Phys. 10 (2010) 026.

[40] O. Pujolas, I. Sawicki, and A. Vikman, The imperfect fluid behind kinetic gravity braiding, J. High Energy Phys. 11 (2011) 156.

[41] L. Lewin, Polylogarithms and Associated Functions (Elsevier Science Ltd, Amsterdam, 1982), ISBN 9780444005502.

[42] L. D. Landau and E. M. Lifishitz, Statistical Physics, Part I (Elsevier, New York, 1980). 